\title{
Characterization and Electro-Optical Properties of the Homopolymer and Copolymers of Ferroelectric Side-Chain Liquid Crystalline Polysiloxanes Containing Two Chiral Center Side Groups
}

\author{
Jenn Chiu Hwang ${ }^{\dagger}$ and Yu Chyi Tsai \\ Department of Chemical Engineering, Yuan Ze University, \\ Taoyuan, Taiwan 32026, Republic of China
}

(Received June 5, 1997)

\begin{abstract}
Aggregation and electro-optical effects in the chiral smectic $\mathrm{C}$ phase of the ferroelectric liquid crystalline homopolymer and two copolymers were investigated. The smectic layers of homopolymer and copolymer were constructed by overlapping about 1.2 and 1.4 times the length of the side-chain mesogenic groups, respectively. The transient scattering mode was obtained by repeated voltage polarity reversal. Ferroelectric liquid crystalline polysiloxanes (FLCPs) exhibit large spontaneous polyarization (Ps) and Ps of homopolymers are larger than those of copolymers. The response times of homopolymers are longer than that of copolymers due to high viscosity. Reversible and bistable transparent-opaque change was successfully realized for the chiral smectic $\mathrm{C}$ phase upon applications of dc electric fields with a response time of several milliseconds at room temperature.

KEY WORDS Electro-Optical Effect / Ferroelectric / Liquid Crystalline Polymer / Spontaneous Polarization /
\end{abstract}

Low molecular weight liquid crystals (LCs) with chiral smectic $\mathrm{C}$ phase have been widely studied because of their ferroelectric properties, electro-optical effects with fast switching time and memory. ${ }^{1-6}$ These features make them particulary suitable for display and light valve devices.

For a display the ferroelectric liquid crystal (FLC) materials should show a chiral smectic $C$ phase over a wide range of temperature including room temperature. The liquid crystal material is required to possess a large spontaneous polarization (Ps). Consequently, a FLC-related device can be operated at reduced driving voltage. These properties are influenced by the molecular structures of liquid crystal compounds. It is interesting to synthesize FLC compounds and compositions which exhibit favorable Ps and exhibit smectic character over a wide temperature range, especially at room temperature. Shibaev et al. synthesized FLCPs which exhibit desirable mechanical properties of polymers and still maintain electro-optical properties of low molecular weight FLC. ${ }^{7}$ This polymer, possessing high speed response and memory effect, has recently attracted much attention. ${ }^{8,9}$ Various side chain liquid crystalline polymers (LCPs) exhibiting a chiral smectic phase and their ferroelectric properties have been reported. ${ }^{10-14}$ However, pure LCPs have some difficulty in the preparation of a uniform cell with macroscopic order necessary for gaining good memory. The response time of LCPs to external stimulation, i.e., electric or magnetic field, is longer than that of LCs because LCPs, in mesophase, being more viscous than LCs. LCP/LC mixtures, in which LC serves as solvent or diluent for LCP, have been studied for reducing the vicosity of LCP. ${ }^{15-17}$ To reduce the viscosity of LCPs, we have synthesized new FLC copolymers with large dielectric constant and a large Ps. ${ }^{18}$

This paper studies the aggregation of the homopoly-

† To whom correspondence should be addressed. mer and copolymer of ferroelectric side chain liquid crystalline polysiloxanes containing 4-(2-chloro-3-methyl pentanoyl)-4'-alkyloxybiphenyl side group. The electrooptical effects based on light scattering and transparent states in wind and unwind helicoidol structures of these polymers are reported.

\section{EXPERIMENTAL}

\section{Materials}

The chemical structures of the side chain ferroelectric liquid crystalline polysiloxanes (FLCPs) are shown in Figure 1. FLCPs used in this study belong to polymethylsiloxane $\left(M_{n}=2270\right)$ and copolymethylsiloxane $\left(M_{n}=1000\right)$ containing 4-alkyloxybiphenyl-4'-(2S,3S)-3methyl-2-chloropentanoate side groups. They are prepared in the same manner as in the literature. ${ }^{19}$ Poly[(2S,3S)-3-methyl-chloropentanoic acid-4',4"-undecyloxy biphenyl ester) methylsiloxane] (HP3M2CPUOB)

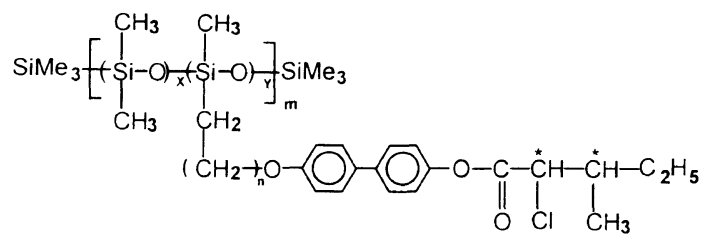

(1) $X: Y=50: 50 \quad m=30$

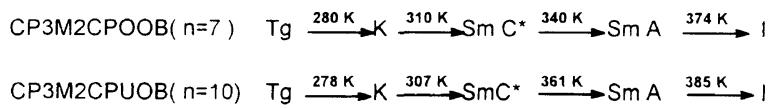

(2) $X: Y=0: 100 \quad m=30$ HP3M2CPUOB $(n=10) \quad T g \stackrel{290 K}{\longrightarrow} \mathrm{K} \stackrel{328 \mathrm{~K}}{\longrightarrow} \mathrm{SmC}^{*} \stackrel{383 \mathrm{~K}}{\longrightarrow} \mathrm{SmA} \stackrel{427 \mathrm{~K}}{\longrightarrow}$ 1

Figure 1. The chemical structures of liquid crystalline homopolymer and copolymers. 
(alkyl spacer $=11)$ is homopolymer, while poly $[(2 S, 3 S)$ 3-methyl-chloropentanoic acid-4",4"-octyloxy biphenyl ester) methylsiloxane] (CP3M2CPOOB) (alkyl spacer $=$ 8 ) and CP3M2CPUOB are copolymers.

\section{Characterization}

A Seiko SSC $5200 \mathrm{H}$ differential scanning calorimetry (DSC) was used to determine thermal transition. The transition temperatures were obtained from specified second heating and cooling scans. DSC heating and cooling rates were $5 \mathrm{~K} \mathrm{~min}^{-1}$. A Nikon FXA polarized optical microscopy (POM) equipped with a Mettler FP82 hot stage was used to observe thermal transition and analyze anisotropy textures. POM heating and cooling rates were $1 \mathrm{~K} \mathrm{~min}^{-1}$. Temperature was adjusted to within $\pm 0.1 \mathrm{~K}$. X-Ray diffraction patterns were observed with a Riguku R-Axis IIC powder diffractometer. The monochromatized X-ray beam from Nikel-filtered $\mathrm{Cu}-K_{\alpha}$ radiation with a wavelength of $0.1542 \mathrm{~nm}$ and temperature controlled sample holder were used.

Ps was evaluated by measuring polarization current upon applying a triangular shaped voltage. ${ }^{20}$ The sample for electro-optical study was sandwiched between two transparent ITO-glass electrodes separated by a poly(ethylene terephthalate) (PET) spacer $(14 \mu \mathrm{m})$. Change in transmission intensity of a $\mathrm{He}-\mathrm{Ne}$ laser light (632.8 $\mathrm{nm}$ ) through the cell without any optical polarizer was detected by a photodiode and recorded by a digital storage oscilloscope.

\section{RESULTS AND DISCUSSION}

To clarify the conformation of FLCP phase, X-ray diffraction studies were carried out over the entire temperature range. Figure 2 shows temperature dependent X-ray diffraction diagrams of homopolymer HP3M2CPUOB. The peaks corresponding to $d$-spacing of the smectic orientation of mesogenics were observed at small angles for all temperatures. In curve $\mathrm{A}$, a sharp first order reflection at $33.43 \AA$ was observed and ascribed to the interplanar distance of the smectic layers. The second order reflection was observed at $17 \AA$. Since the reciprocal of these two spacings is $1: 2$, the experimental results in Figure 3 verify the formation of a smectic texture. The fan-shape texture is a characteristic for a smectic A phase, as confirmed by POM observation. The diffraction peaks in the small angular region shifted slightly in the vicinity of the phase transition temperature. When the temperature was lowered from $388 \mathrm{~K}$ to $330 \mathrm{~K}$, $d$-spacing of the first order reflection decreased from $33.43 \AA$ to $32 \AA$. This is evidence for the formation of the tilted chiral smectic $C$ phase. A diffuse reflection was observed at a wide $\mathrm{Bragg}$ angular region of about $4.7 \AA$, which corresponds to the lateral spacing of two mesogenic side groups. The molecular length of the side-chain FLC calculated assuming the most extended conformation using the Corey-Pauling-Koltum (CPK) molecular model to be $31 \AA$. The layer spacing of the side group FLC in the smectic A phase evaluated from the X-ray diffraction was only $28.1 \AA, 3 \AA$ less than the calculated value. ${ }^{21}$ A $3 \AA$ length difference may originate from the conformationally disordered structure of molecules. If the mesogenic side group is placed in

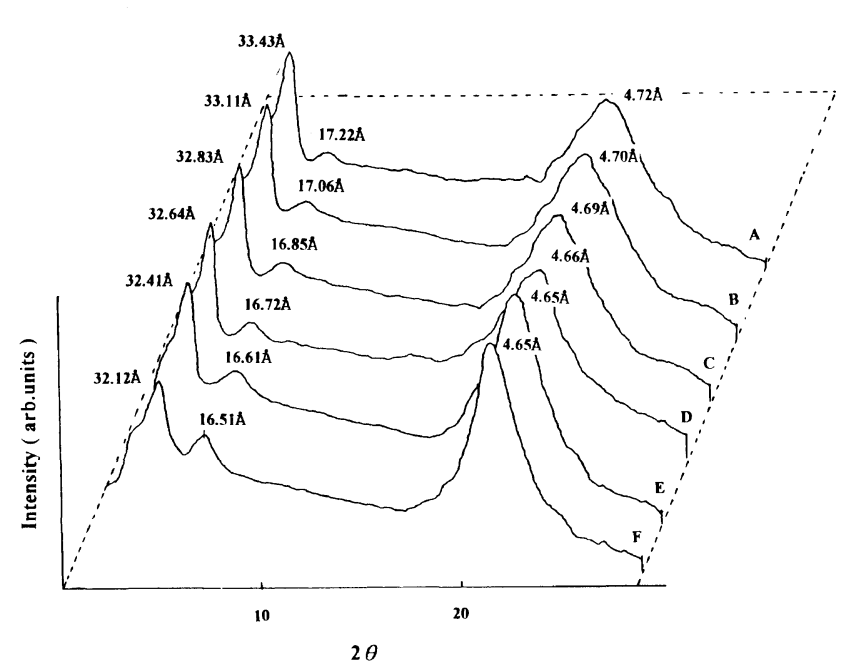

Figure 2. Temperature dependence of $\mathrm{X}$-ray diffraction diagrams of HP3M2CPUOB at (A) 388, (B) 380, (C) 365, (D) 352, (E) 340, and (F) $330 \mathrm{~K}$.

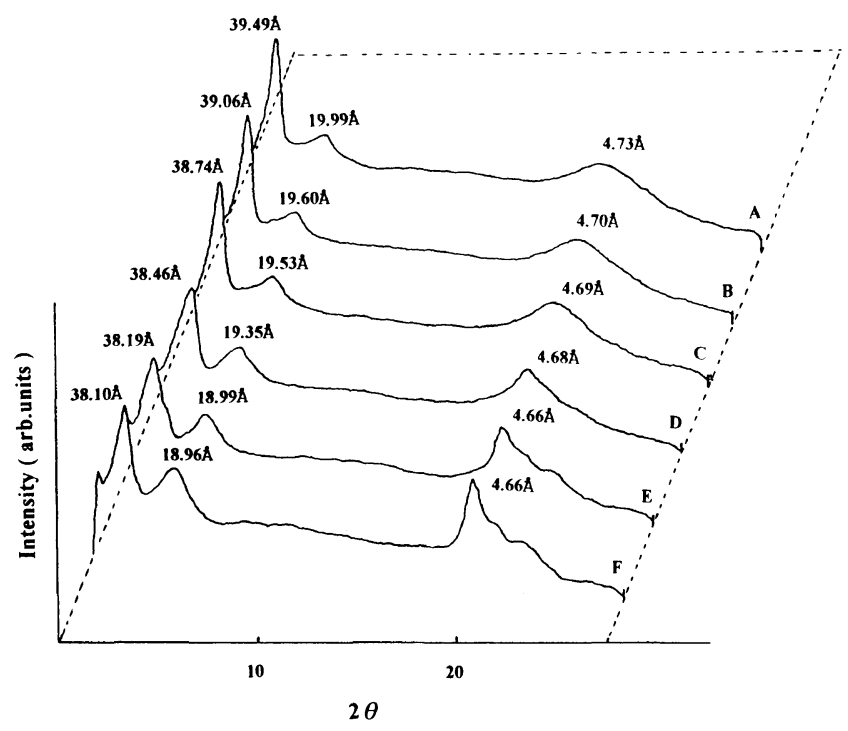

Figure 3. Temperature dependence of X-ray diffraction diagrams of CP3M2CPUOB at (A) 363, (B) 360, (C) 354, (D) 348, (E) 342, and (F) $320 \mathrm{~K}$

same side on the main chain, the layer spacing of HP3M2CPUOB in the smectic A phase should be about $28.1 \AA$. The $d$-spacing of smectic A for HP3M2CPUOB is $33.43 \AA$, longer than the measured length of $28.1 \AA$ for the side group. Therefore, it is suggested that the mesogenic side groups with structurally symmertrical smectic layers are formed on the opposite side of the main chain. The smectic layers of the homopolymer were constructed by overlapping about 1.2 times the length of a mesogenic side group.

Figure 3 presents the temperature dependent X-ray diffraction diagrams of CP3M2CPUOB. A sharp reflection at small angles (associated with the smectic layers) and broad reflection at wide angles (associated with the lateral packing) are shown in all curves. Curve A shows a sharp first order reflection at $39.49 \AA$ which corresponds to smectic layers and diffuse reflection at about $4.7 \AA$ which corresponds to lateral spacing of two mesogenic side groups. The smectic A phase of CP3M2CUOB was an interlayer about 1.4 times the 


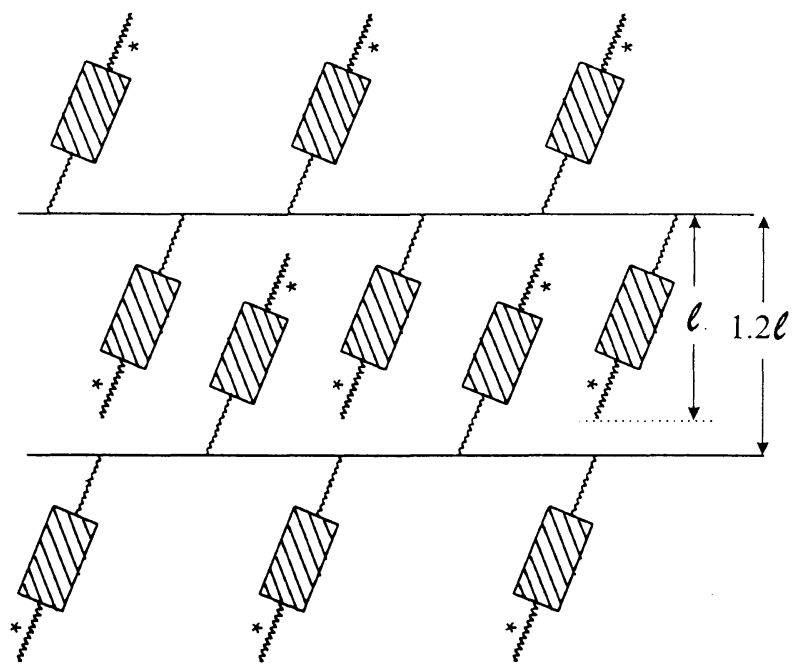

(a)

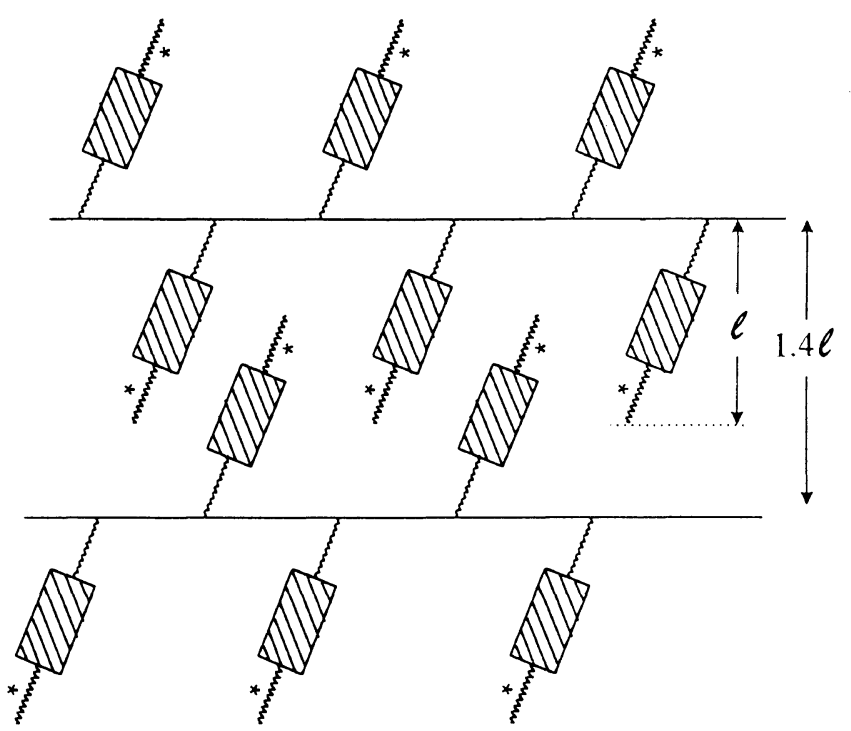

(b)

Figure 4. Schematic illustration of layer aggregation for (a) homopolymer and (b) copolymer.

measured length of $28.1 \AA$. If the mesogenic groups stand vertically in the layer sheets, the side chain length should be $39.49 \AA / 2=19.75 \AA$. This value is too short and contradicts the above measured length of $28.1 \AA$. Therefore, we may assume that the layers are formed by an overlap of $16.5 \AA$ of two side groups. These differences indicate that HP3M2CPUOB and CP3M2CPUOB form an overlapping head-to-head molecular association. When the measuring temperature was lowered form $363 \mathrm{~K}$ to $310 \mathrm{~K}, d$-spacing of the first order reflection decreased from $39.49 \AA$ to $38 \AA$. This is the strong evidence for the formation of the chiral smectic C phase.

As indicated above, the mesogenic group is not full-overlapping. Figure 4 shows the schematic illustration of layer models of homopolymer and copolymer. It is reasonable to suggest that $d$-spacings (smectic layer) of homopolymer HP3M2CPUOB are about 1.2 times the length of the side chain mesogenic group (see Figure $4 a)$. The $d$-spacings (smectic layer) of copolymer CP3M2CPUOB about 1.4 times the length of the side

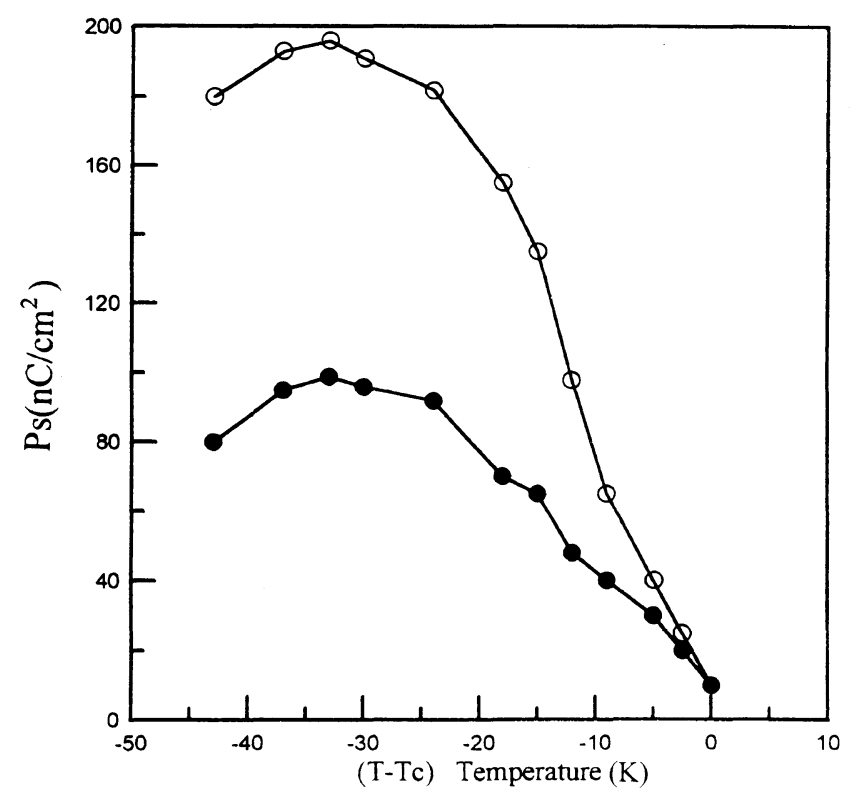

Figure 5. Temperature dependence of spontaneous polarization for homopolymer HP3M2CPUOB $(O)$ and copolymer CP3M2CPUOB

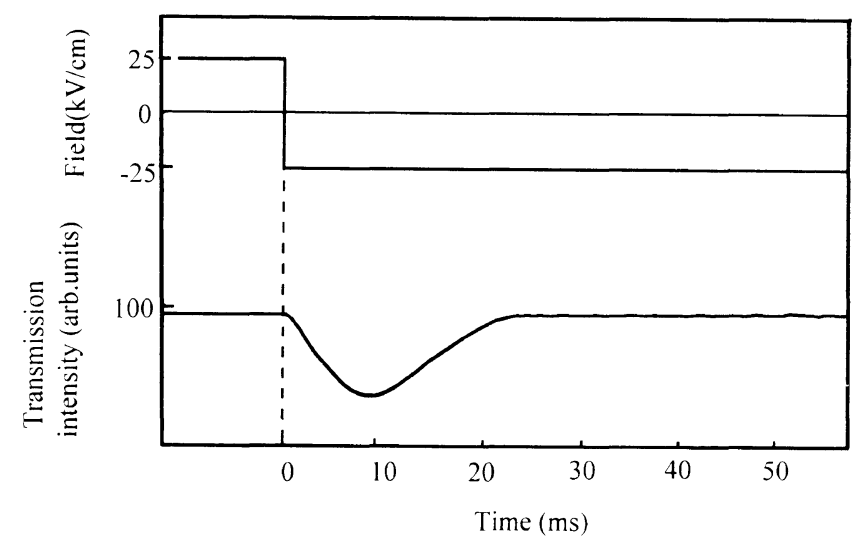

Figure 6. Applied voltage waveform and waveform of transmission intensity response to polarity reversal of applied electric field for HP3M2CPUOB at $T-T_{\mathrm{c}}=-16 \mathrm{~K}$. The applied voltage was $50 \mathrm{kV} \mathrm{cm}^{-1}$ and the cell thickness was $14 \mu \mathrm{m}$.

chain mesogenic group (see Figure 4b).

The temperature dependence of Ps in homopolymer HP3M2CPUOB and copolymer CP3M2CPUOB are shown in Figure 5. Large Ps were observed for both FLCPs. Ps increased with decreasing temperature in the chiral smectic $\mathrm{C}$ phase for both FLCPs. The largest Ps of HP3M2CPUOB and CP3M2CPUOB were 196 and $95 \mathrm{nC} \mathrm{cm}^{-2}$, respectively. The homopolymer has a larger Ps than that of copolymer due to decrease in mesogenic group content, which would consequently reduce the Ps of FLCP. Ps of FLCPs decreased at low temperature for both FLCPs. This may be associated with the increased viscosity in FLCP at low temperature. For high viscosity, the applied field strength may not be sufficient to completely induce reorientation of the Ps of the molecules.

Figure 6 shows the response waveform of light transmission intensity to an applied polarity reversal in HP3M2CPUOB. The light transmission intensity decreased remarkably with application of electric field polarity reversal in a certain time interval. The 


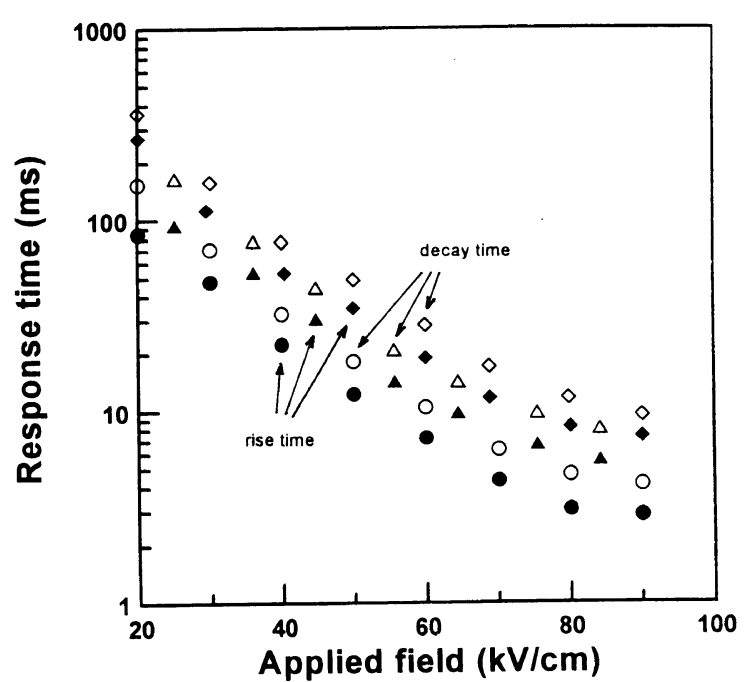

(a)

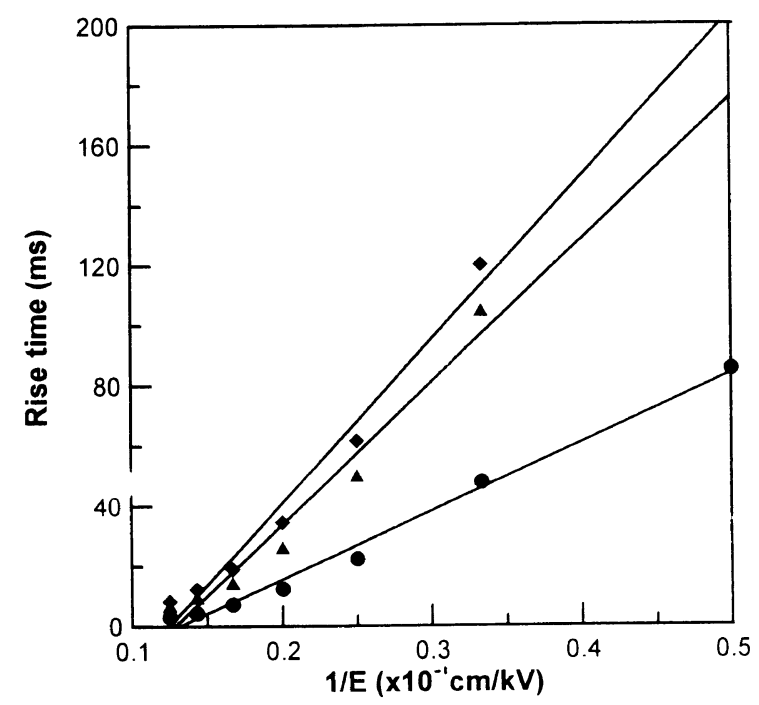

(b)

Figure 7. (a) Applied field dependence of rise time and decay time of the TSM effect at $T-T_{\mathrm{c}}=-26 \mathrm{~K}$. The cell thickness was $14 \mu \mathrm{m}$. $(\diamond, \diamond)$ HP3M2CPUOB; $(\triangle, \Delta)$ CP3M2CPUOB; $(\bigcirc, 0)$ CP3M2CPOOB. (b) Dependence of rise time on the reciptocal of applied field at $T-T_{\mathrm{c}}=-26 \mathrm{~K}$. ( $\bullet$ HP3M2CPUOB; (A) CP3M2CPUOB; (O) CP3M2CPOOB.

transmittance exhibits an instantaneous dip state. This dip state is due to light scattering at the instant of transmission between two ferroelectric uniform states, and this electrooptical effect is called the TSM effect. ${ }^{22}$ Figure $7 \mathrm{a}$ shows the applied field dependence of the response time for the electro-optical effect in homopolymer HP3M2CPUOB (spacer $=11)$, copolymer CP3M2CPUOB (spacer $=11)$, and $\mathrm{CP} 3 \mathrm{M} 2 \mathrm{CPOOB}$ $($ spacer $=8)$. Rise and decay times are defined as response times for the transmission intensity to change from $90 \%$ to $10 \%$ and $10 \%$ to $90 \%$, respectively. The response time became shorter with increasing applied field. Figure $7 \mathrm{~b}$ shows the dependence of rise time on the reciprocal of applied field at various temperatures. The rise time is inversely proportional to the applied field. Figure $7 \mathrm{~b}$ shows the dependence of rise time on induced by Ps with the electric field. The rise time also became longer with decreasing temperature. The response time of homopolymer HP3M2CPUOB is longer 608

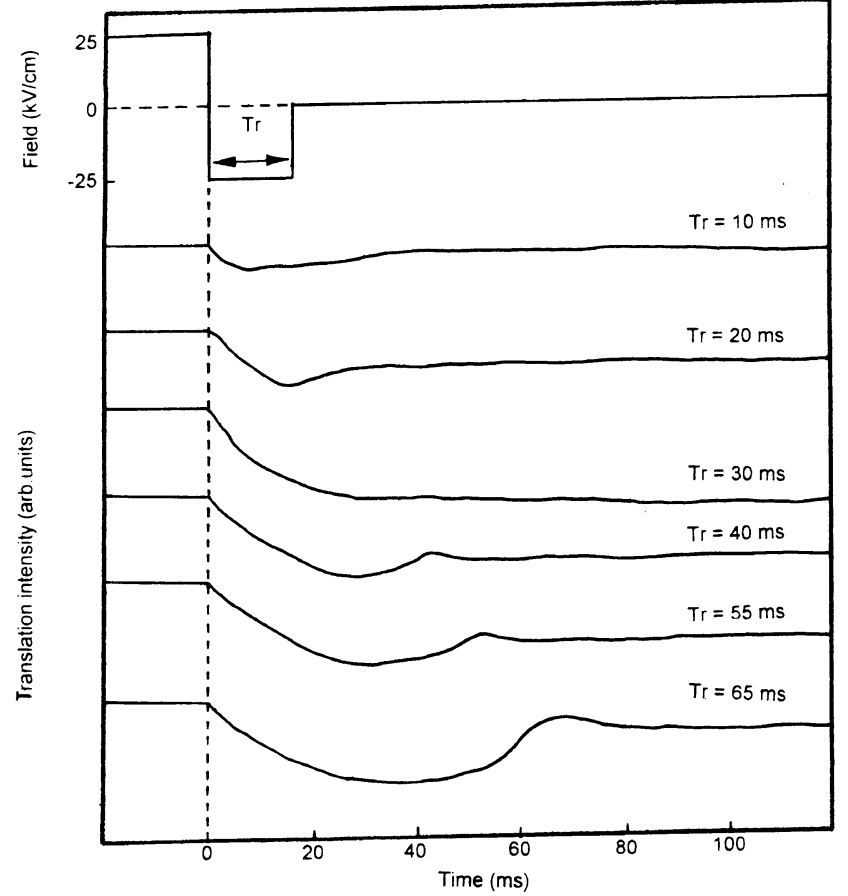

Figure 8. Applied field waveform and response waveform of transmission intensity to applied pulse field of reversal polarity for the period of $T_{\mathrm{r}}$ in CP3M2CPUOB at $T-T_{\mathrm{c}}=-26 \mathrm{~K}$. The cell thickness was $14 \mu \mathrm{m}$.

than those of copolymer CP3M2CPUOB. The response time should be related to the magnitude of Ps and viscosity. Generally, the viscosity and Ps increased with decreasing temperature for FLC. Therefore, the influence of increased viscosity at low temperature may have more pronounced effect than increase of Ps. Due to the viscosity effect, the relaxation times for the reorientations of Ps and polymer main chains increased with decreasing temperature. The copolymer has lower viscosity due to the dilution of the mesogenic group on the copolymer backbone. For lower viscosity, the orientation of copolymer molecules is easily controlled by applying an electric or magnetic field.

The response waveforms of transmission intensity obtained for CP3M2CPUOB(spacer $=11$ ) and CP3M$2 \mathrm{CPOOB}$ (spacer $=8$ ) upon applying the electric field pulse are shown in Figures 8 and 9. The transparent state was obtained due to a uniform domain of an unwinding state when the dc field was imposed. However, the transparent state of FLCPs turned to opaque state due to the stripe patterns when the pulse field of reversal polarity was applied. For the pulse field with $T_{\mathrm{r}}=$ $30 \mathrm{~ms}$ in CP3M2CPUOB as shown in Figure 8, the opaque state could be maintained even after reversal polarity was turned off. This suggests that the stripe pattern observed by POM which appeared at the instant of polarization reversal was caused by the helical structure. From the results of Figure 8, it can be seen that the transparent and opaque states are realized under dc voltage and repeated polarity reversal of voltage, respectively. A shorter period of pulse field with $T_{\mathrm{r}}=22 \mathrm{~ms}$ in CP3M2CPOOB is necessary for gaining good memory. The relaxation time for reorientation of Ps and main chain is longer than for the present FLCP with longer spacer than that with short spacer. In this 


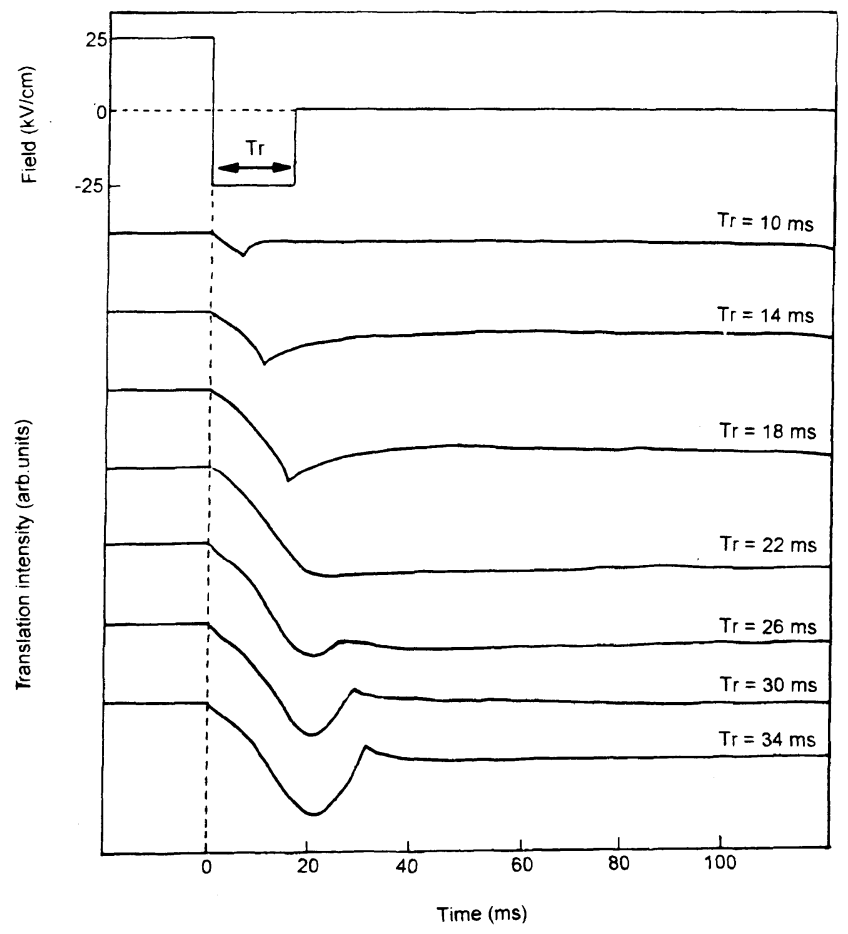

Figure 9. Applied field waveform and response waveform of transmission intensity to applied pulse field of reversal polarity for the period of $T_{\mathrm{r}}$ in CP3M2CPOOB at $T-T_{\mathrm{c}}=-26 \mathrm{~K}$. The cell thickness was $14 \mu \mathrm{m}$.

study, the ITO glass surface was not specially treated by rubbing and optical polarizer was not necessary. The switching time between transparent and opaque states was several ms. The operation time of FLCP is shorter than that of the conventional system which must be heated to isotropic and then cooled with electric field to the mesophase which exists at low temperature. This FLCP system in chiral smectic $\mathrm{C}$ phase thus has the potential for application to a light valve device and storage device utilized for rewriting optical information.

\section{CONCLUSION}

The FLCP homopolymer and copolymers with large Ps were newly synthesized and their structures were clarified. The smectic layers of FLCPs are constructed by partial overlapping of side groups. The smectic layers of homopolymer and copolymer are 1.2 and 1.4 times the length of the side mesogenic groups, respectively. The relaxation times for the reorientations of Ps and polymer main chains increased with decreasing temperature. The response time of copolymer is shorter than that of homopolymer due to lower viscosity. The influence of viscosity increase at low temperature has a more pronounced effect than the increase of Ps. Therefore, the response time of copolymer FLCP with shorter spacer is shorter than that of homopolymer. The reversible and bistable transparent-opaque change was successfully realized by dc voltage and polarity reversal of voltage, respectively. The transparent and opaque states were maintained stably, even though an electric field was turned off. FLCP exhibits high speed response times of several milliseconds.

Acknowledgment. The authors thank the National Science Council of the Republic of Chain for financial support of this work under grant NSC86-2216-E155-004.

\section{REFERENCES}

1. R. B. Meyer, L. Liebert, L. Strzelecki, and P. Keller, J. Phys. (Paris) Lett., 36, L69 (1975).

2. K. Yoshino, K. G. Balakrishnan, T. Uemoto, Y. Iwasaki, and Y. Inuishi, Jpn. J. Appl. Phys., 17, 597 (1975).

3. N. A. Clark and S. T. Lagerwall, Appl. Phys. Lett., 36, 899 (1980).

4. K. Yoshino and M. Ozaki, Jpn. Appl. Phys., 23, L385 (1984).

5. M. Ozaki, S. Kishio, and K. Yoshino, Mol. Cryst. Liq. Cryst., 146, 251 (1987).

6. M. Ozaki, S. Kishio, K. Yoshino, N. Mikami, R. Higuchi, and T. Sakurai, Jpn. J. Appl. Phys., 26 (Suppl.), 110 (1987).

7. V. P. Shibaev, M. V. Kozlovsky, L. A. Beresnev, L. M. Blinov, and N. A. Platé, Polym. Bull., 12, 299 (1984).

8. R. Zental, G. Reckert, and B. Reck, Liquid Crystals, 2, 83 (1987).

9. D. S. Parmar, N. A. Clark, P. Keller, D. M. Walba, and M. D. Wand, J. Phys. (Paris), 51, 355 (1990).

10. V. P. Shibaev, M. V. Kozlovsky, N. A. Platé, L. A. Beresnev, and L. M. Blinov, Liquid Crystals, 8, 545 (1990).

11. S. U. Vallerien, F. Kremer, and E. W. Fischer, Macromol. Chem. Rap. Commun., 11, 593 (1990).

12. H. Ento, S. Hachiya, S. Uchida, K. Hashimoto, and K. Kawasaki, Liquid Crystals, 9, 635 (1991).

13. H. Kapizta and R. Zental, Macromol. Chem., 192, 1859 (1991).

14. G. H. Hsiue, C. H. Hsu, and L. J. Shin, Macromolecules, 26, 3161 (1993).

15. M. S. Sefton and H. J. Coles, Polymer, 26, 1319 (1985).

16. T. Kajiyama, H. Kikuchi, A. Miyamoto, S. Moritomi, and J. C. Hwang, Chem. Lett., 817 (1989).

17. J. C. Hwang, H. Kikuchi, and T. Kajiyama, Polymer, 33, 1822 (1992).

18. G. H. Hsiue, R. H. Lee, and J. C. Hwang, Polymer, 37, 1095 (1996).

19. G. H. Hsiue, P. J. Hsieh, S. L. Wu, and C. S. Hsu, Polym. Bull., 33, 159 (1994).

20. K. Miyasato, S. Abe, H. Takezoe, A. Fukuta, and E. Kuze, Jpn. J. Appl. Phys., 22, L661 (1983).

21. J. C. Hwang, M. C. Shu, H. Moritake, and T. Yoshino, Macromol. Chem. Phys., 198, 1293 (1997).

22. J. C. Hwang, Y. Fuwa, H. Moritake, H. S. Gu, M. Ozaki, and K. Yoshino, Jpn. J. Appl. Phys., 34, L560 (1995). 